\title{
A New Ranking Method for Interval-Valued Intuitionistic Fuzzy Numbers and Its Application in Multi-Criteria Decision-Making
}

\author{
Jeevaraj Selvaraj ${ }^{1, *([)}$ and Abhijit Majumdar ${ }^{2}$ (i) \\ 1 Department of Mathematics, Atal Bihari Vajpayee Indian Institute of Information Technology and \\ Management, Gwalior 474015, India \\ 2 Department of Textile and Fibre Engineering, Indian Institute of Technology Delhi, New Delhi 110016, India; \\ majumdar@textile.iitd.ac.in \\ * Correspondence: jeevaraj@iiitm.ac.in
}

Citation: Selvaraj, J.; Majumdar, A. A New Ranking Method for Interval-Valued Intuitionistic Fuzzy Numbers and Its Application in Multi-Criteria Decision-Making. Mathematics 2021, 9, 2647. https:// doi.org/10.3390/math9212647

Academic Editors: James Liou and Artūras Kaklauskas

Received: 26 September 2021

Accepted: 18 October 2021

Published: 20 October 2021

Publisher's Note: MDPI stays neutral with regard to jurisdictional claims in published maps and institutional affiliations.

Copyright: (c) 2021 by the authors. Licensee MDPI, Basel, Switzerland. This article is an open access article distributed under the terms and conditions of the Creative Commons Attribution (CC BY) license (https:/ / creativecommons.org/licenses/by/ $4.0 /)$.

\begin{abstract}
Ranking of interval-valued intuitionistic fuzzy numbers (IVIFNs) is an important task for solving real-life Decision-Making problems. It is a potential area of research that has attracted the researchers working in fuzzy mathematics. Researchers worldwide are looking for a unique ranking principle that can be used to discriminate any two arbitrary IVIFNs. Various ranking functions on the set of IVIFNs have been proposed. However, every method has some drawbacks in ranking arbitrary IVIFNs due to the partial ordering. This paper introduces a new ranking principle for comparing two arbitrary IVIFNs by defining a new score function based on the non-membership value of IVIFNs. In this paper, firstly, the limitations of a few well-known and existing ranking methods for IVIFNs have been discussed. Secondly, a new non-membership score on the class of IVIFNs has been introduced. Thirdly, the superiority of the proposed score function in ranking arbitrary IVIFNs over the existing methods has been demonstrated. Finally, the proposed non-membership score function has been utilized in interval-valued intuitionistic fuzzy TOPSIS (IVIF-TOPSIS) using numerical examples.
\end{abstract}

Keywords: interval-valued intuitionistic fuzzy number; non-membership score function; MCDM; IVIF-TOPSIS; IVIF-MCDM; defuzzification

\section{Introduction}

Decision-Making problems often involve imprecise information that can be modelled better using fuzzy sets than classical sets. The main advantage of using fuzzy sets is that they give freedom to the decision-maker to assign membership values between 0 and 1 , whereas in the case of crisp sets, decision-makers are restricted to providing a crisp value (either 0 or 1 ). However, in most of the real-life problems, incompleteness occurs in the data in addition to imprecision. Intuitionistic fuzzy set (IFS) was introduced in the literature as a generalization of the classical fuzzy set. The main characteristics of an IFS is that the sum of the degrees of membership and non-membership values of any element in the IFS will be less than or equal to 1. Further, it has been generalized to interval-valued intuitionistic fuzzy set (IVIFS) since the IVIFSs model incompleteness in a better way than the real-valued IFSs. The ranking of IVIFSs will play an essential role in solving any decision-making problem involving imprecision and incompleteness. A lot of ranking procedures have been introduced in the literature for differentiating any two arbitrary IVIFNs. $\mathrm{Xu}$ [1] introduced the idea of score and accuracy functions on the set of IVIFNs. Using these two functions, he tried to compare arbitrary IVIFNs. His "score function" was nothing but the difference between the mid-points of the membership and non-membership functions, and the "accuracy function" was the sum of the midpoints of the membership and non-membership functions. However, if $A=([a, b][c, d])$ 
and $B=([a-\epsilon, b+\epsilon][c-\epsilon, d+\epsilon])$ are any two IVIFNs, then their score and accuracy functions are equal, which shows the limitation Xu's ranking procedure. In the year 2009, Ye [2] pointed out few drawbacks of other existing ranking methods on the set of realvalued IFS and proposed a novel accuracy score function on IVIFNs. The proposed score function ([2]) was used in solving Multi-Criteria Decision-Making (MCDM) problems modelled with IVIFNs. A non-membership score function on the set of IVIFNs was introduced by Nayagam and Geetha [3]. They claimed that the proposed non-membership score function overcomes the previous drawbacks. However, their score function also ranked two different IVIFNs as equal in some cases. Further, the limitations of the nonmembership score function were identified by the same group. They improved the ranking of IVIFNs by introducing an axiomatic set of score functions [4]. Bai [5] proposed an improved score function for ranking IVIFNs, and claimed that the proposed method overcomes the drawbacks of earlier methods. Unfortunately, this improved score function was also not able to discriminate arbitrary IVIFNs. In 2016, Garg [6] proposed a new ranking principle on the set of IVIFNs for comparing arbitrary IVIFNs by generalizing Bai's [5] improved score function. He claimed that his method provides better results when it is compared with other methods $[1,2,5,7]$. However, his method also failed to rank arbitrary IVIFNs and also had the same drawbacks of improved score function [5] since it is the generalization of Bai's [5] improved score function. Sahin [8] proposed another score function called the improved accuracy score of an IVIFN and showed that the improved accuracy score function ranks arbitrary IVIFNs more effectively than the familiar ranking methods. He also utilized the improved accuracy function in improving the Decision-Making algorithm. Fangwei and Xu [9] identified the illogicalities of Sahin's improved accuracy function by using illustrative examples and introduced a new ranking principle that can overcome the drawbacks of Sahin's ranking procedure. However, their method also had some other disadvantages in discriminating two different IVIFNs, which was identified by Nayagam et al. [10]. Nayagam et al. [10] proposed a non-hesitance score on the set of IVIFNs and discussed its advantages using various illustrative examples. In addition to that, they [10] identified the new subclass of IVIFNs where the non-hesitance score function defines total ordering.

IVIFS can be utilized in solving many real-life problems from different domains [11-13]. Researchers have utilized IVIFNs in pattern recognition, clustering and image processing, and so forth. Jeevaraj [11] proposed a new similarity measure on the class of IVIFS by using the non-hesitance score function [10] and utilized the proposed similarity measure in the field of pattern recognition. Zhang and Yu [14] introduced a new Multi-Attribute Decision-Making (MADM) algorithm by using the idea of cross-entropy and generalized the TOPSIS [15] method with IVIFNs.

Based on the ongoing discussion of the different ranking procedures of IVIFNs, it can be understood that there is no reliable method that can be used to compare arbitrary IVIFNs. This provides a pathway for introducing a new score function for comparing arbitrary IVIFNs. In this research, a new score function is being propounded for comparing arbitrary IVIFNs based on their non-membership value. The main aim of this work is to introduce a new and reliable ranking principle for comparing two different IVIFNs. In this paper, we:

1. discuss the limitations of a few well-known ranking methods for IVIFNs;

2. introduce a new non-membership score on the class of IVIFNs and study its mathematical properties;

3. demonstrate the superiority of the proposed score function, over the existing score functions, in ranking arbitrary IVIFNs;

4. utilize the proposed score function in interval-valued intuitionistic fuzzy TOPSIS (IVIF-TOPSIS);

5. explain the applicability of the proposed ranking method in solving interval-valued intuitionistic fuzzy multi-criteria decision-making (IVIF-MCDM) problems using numerical examples. 
The remainder of the paper is organized in the following manner. After the introduction, a few basic definitions are presented in Section 2. Section 3 discusses the various drawbacks of existing methods in ranking arbitrary IVIFNs. Section 3 also compares different available ranking principles of IVIFNs. Section 4 presents the new score function based on the non-membership value of an IVIFN, along with its essential mathematical properties. Section 4 also encompasses the comparison between the proposed score function and the available ranking procedures of IVIFNs. Section 5 presents the proposed score function's applicability in solving the MCDM problem modelled under an intervalvalued intuitionistic fuzzy environment. Numerical illustrations of the proposed MCDM algorithm are also given in Section 5. Finally, conclusions are presented in Section 6.

\section{Preliminaries}

Some basic definitions are given in this section.

Definition 1 (Xu 2007, [1]). Let $D[0,1]$ be the set of all closed subintervals of the interval $[0,1]$. An IVIFS on a set $X \neq \phi$ is an expression given by $A=\left\{\left\langle x, \mu_{A}(x), v_{A}(x)\right\rangle: x \in X\right\}$, where $\mu_{A}: X \rightarrow D[0,1], v_{A}: X \rightarrow D[0,1]$ with the condition $0<\sup _{x} \mu_{A}(x)+\sup _{x} v_{A}(x) \leq 1$.

The intervals $\mu_{A}(x)$ and $v_{A}(x)$ denote, respectively, the degree of belongingness and non-belongingness of the element $x$ to the set $A$. Thus, for each $x \in X, \mu_{A}(x)$ and $v_{A}(x)$ are closed intervals whose lower and upper end points are, respectively, denoted by $\mu_{A_{L}}(x), \mu_{A_{U}}(x)$ and $v_{A_{L}}(x), \mu_{A_{U}}(x)$. We denote $A=\left\{\left\langle x,\left[\mu_{A_{L}}(x), \mu_{A_{U}}(x)\right],\left[v_{A_{L}}(x), v_{A_{U}}(x)\right]\right\rangle: x \in X\right\}$ where $0<\mu_{A_{U}}(x)+v_{A_{U}}(x) \leq 1$.

For each element $x \in X$, we can compute the unknown degree (hesitance degree) of belongingness $\pi_{A}(x)$ to $A$ as $\pi_{A}(x)=1-\mu_{A}(x)-v_{A}(x)=\left[1-\mu_{A_{U}}(x)-v_{A_{U}}(x), 1-\right.$ $\left.\mu_{A_{L}}(x)-v_{A_{L}}(x)\right]$. An intuitionistic fuzzy interval number (IFIN) is denoted by $A=([a, b],[c, d])$ for convenience.

Definition $2(\mathrm{Xu} 2007,[1])$. Let $A=([a, b],[c, d])$ be an IVIFN. Then, the score function $S$ of $A$ and an accuracy function $H$ of $A$ are defined, respectively, as follows $S(A)=\frac{a+b-c-d}{2}$ and $H(A)=\frac{a+b+c+d}{2}$.

Definition 3 (Ye 2009, [2]). Let $A=([a, b],[c, d])$ be an IVIFN. Then the novel accuracy score function $M$ of $A$ is defined as: $M(A)=\frac{a-(1-a-c)+b-(1-b-d)}{2}, M(A) \in[-1,1]$.

Definition 4 (Nayagam et al. 2011, [7]). Let $A=([a, b],[c, d])$ be an IVIFN. Then the accuracy score function $L$ of $A$ is defined as: $L(A)=\frac{a+b-d(1-b)-c(1-a)}{2}, L(A) \in[-1,1]$.

Definition 5 (Bai 2013, [5]). Let $A=([a, b],[c, d])$ be an IVIFN. Then, the improved score function $I$ of $A$ is defined as: $I(A)=\frac{a+a(1-a-c)+b+b(1-b-d)}{2}, I(A) \in[0,1]$.

Definition 6 (Garg 2016, [6]). Let $A=([a, b],[c, d])$ be an IVIFN. Then, the generalized improved score function GIS of $A$ is defined as: $\operatorname{GIS}(A)=\frac{a+b}{2}+k_{1} a(1-a-c)+k_{2} b(1-b-d)$ with $k_{1}+k_{2}=1$.

Definition 7. Let $A=\left(\left[a_{1}, b_{1}\right],\left[c_{1}, d_{1}\right]\right)$ and $B=\left(\left[a_{2}, b_{2}\right],\left[c_{2}, d_{2}\right]\right)$ be two IVIFNs. $A \subseteq_{1} B$ if $a_{1} \leq a_{2}, b_{1} \leq b_{2}, c_{1} \geq c_{2}$ and $d_{1} \geq d_{2}$.

Definition 8. Let $A=\left(\left[a_{1}, b_{1}\right],\left[c_{1}, d_{1}\right]\right)$ and $B=\left(\left[a_{2}, b_{2}\right],\left[c_{2}, d_{2}\right]\right)$ be two IVIFNs. Then, $A+B$ is defined as $A+B=\left(\left[a_{1}+a_{2}-a_{1} a_{2}, b_{1}+b_{2}-b_{1} b_{2}\right],\left[c_{1} c_{2}, d_{1} d_{2}\right]\right)$. 


\section{Comparison between Various Existing Methods for Ranking IVIFNs}

This section compares different methods for ranking arbitrary IVIFNs and discusses the drawbacks of one method over other methods based on their limitations using numerical examples.

Here, we discuss the limitations of Generalized Improved Score function (GIS) [6] in different cases.

1. (I I ) If $A_{1}=\left([0,0]\left[c_{1}, d_{1}\right]\right)$ and $A_{2}=\left([0,0]\left[c_{2}, d_{2}\right]\right)$ are two IVIFNs with $c_{1} \geq c_{2}$, $d_{1} \geq d_{2}$, then $\operatorname{GIS}\left(A_{1}\right)=\operatorname{GIS}\left(A_{2}\right)=0 \Rightarrow A_{1}=A_{2}$. But it is clear that $A_{1} \neq A_{2}$. Hence the generalized improved score function introduced by Garg [6] fails to rank arbitrary IVIFNs of this type;

2. (I $)$ Let $\alpha_{1}=\left(\left[a_{1}, b_{1}\right],\left[c_{1}, d_{1}\right]\right)$ be an IVIFN. If $a_{1}=b_{1}, c_{1}=d_{1}$ then $\operatorname{GIS}\left(\alpha_{1}\right)=$ $a_{1}+\left(k_{1}+k_{2}\right) a_{1}\left(1-a_{1}-c_{1}\right)=a_{1}\left(2-a_{1}-c_{1}\right)$. This implies that $k_{1}$ and $k_{2}$ do not have any importance in ranking arbitrary intuitionistic fuzzy numbers;

3. (I $\left.I_{3}\right)$ Let $A_{1}=\left(\left[a_{1}, b_{1}\right],\left[c_{1}, d_{1}\right]\right)$ be an IVIFN. If $k_{1}=0, k_{2}=1$, then $\operatorname{GIS}\left(A_{1}\right)=$ $\frac{a_{1}+b_{1}}{2}+b_{1}\left(1-b_{1}-d_{1}\right)$; that is, if $A_{1}=\left(\left[a_{1}, b_{1}\right],\left[c_{1}, d_{1}\right]\right), A_{2}=\left(\left[a_{1}, b_{1}\right],\left[c_{2}, d_{1}\right]\right)$ are any two IVIFNs, then $\operatorname{GIS}\left(A_{1}\right)=\operatorname{GIS}\left(A_{2}\right)=\frac{a_{1}+b_{1}}{2}+b_{1}\left(1-b_{1}-d_{1}\right) \Rightarrow A_{1}=A_{2}$ which is illogical;

4. ( $\left.I_{4}\right)$ Let $A_{1}=\left(\left[a_{1}, b_{1}\right],\left[c_{1}, d_{1}\right]\right)$ be an IVIFN. If $k_{1}=1, k_{2}=0$, then $\operatorname{GIS}\left(A_{1}\right)=$ $\frac{a_{1}+b_{1}}{2}+a_{1}\left(1-a_{1}-c_{1}\right)$; that is, if $A_{1}=\left(\left[a_{1}, b_{1}\right],\left[c_{1}, d_{1}\right]\right), A_{2}=\left(\left[a_{1}, b_{1}\right],\left[c_{1}, d_{2}\right]\right)$ are any two IVIFNs, then $\operatorname{GIS}\left(A_{1}\right)=\operatorname{GIS}\left(A_{2}\right)=\frac{a_{1}+b_{1}}{2}+a_{1}\left(1-a_{1}-c_{1}\right) \Rightarrow A_{1}=A_{2}$. However, $A_{1} \neq A_{2}$. Here, both the initial assumptions contradict each other. Hence, they cannot be considered (together) in the proof.

5. (I $\left.I_{5}\right)$ Let $A_{1}=\left(\left[a_{1}, b_{1}\right],\left[c_{1}, d_{1}\right]\right)$ be an IVIFN. If $a_{1}=k_{2}=0$, then $\operatorname{GIS}(A)=\frac{b_{1}}{2}$. Let $A=\left(\left[0, b_{1}\right],\left[c_{1}, d_{1}\right]\right), B=\left(\left[0, b_{1}\right],\left[c_{2}, d_{2}\right]\right)$ be any two IVIFNs. Then, $\operatorname{GIS}(A)=\frac{b_{1}}{2}=$ $\operatorname{GIS}(B) \Rightarrow A=B$. Here, the GIS function discriminates two arbitrary IVIFNs based on $b_{1}$ (upper value of membership function, irrespective of non-membership values $c_{1}$ and $d_{1}$ ) alone, which is illogical;

6. ( $\left.I_{6}\right)$ For any real number $r=([r, r],[r, r]), \forall r \leq 0.5$, GIS $(r)=2 r(1-r)$. Let $M=\left(\left[r_{1}, r_{1}\right],\left[r_{1}, r_{1}\right]\right)$ and $N=\left(\left[1-r_{1}, 1-r_{1}\right],\left[1-r_{1}, 1-r_{1}\right]\right), \forall r \leq 0.5$ be any two IVIFNs. Then GIS $(M)=G I S(N)=2 r_{1}\left(1-r_{1}\right) \Rightarrow M=N$. This shows that Garg's [6] GIS score function fails to rank arbitrary real numbers.

7. Comparison of Generalized Improved score function (GIS) with Xu's [1] score function: Let $A_{1}=([0,0][0.4,0.5])$ and $A_{2}=([0,0][0.5,0.7])$ be two IVIFNs. Then $\operatorname{GIS}\left(A_{1}\right)=0=\operatorname{GIS}\left(A_{2}\right) \Rightarrow A_{1}=A_{2}$. However $A_{1} \neq A_{2}$ because their nonmembership values are different, and they are non-zero. If Xu's [1] score function is applied, then we get $A_{1}>A_{2}$. In these places, Xu's [1] score function works better which is happening because Garg's method measures the membershipness of an arbitrary IVIFNs, and hence the generalized improved score function maps the IVIFNs with zero membership value to zero;

8. Let $M=\left([0,0],\left[c_{1}, d_{1}\right]\right)$ and $N=\left([0,0],\left[c_{2}, d_{2}\right]\right)=\left([0,0],\left[c_{1}+\epsilon, d_{1}-\epsilon\right]\right), \forall \epsilon \in$ $[0,1], \epsilon<c_{1}$ be any two IVIFNs. Then we get, GIS $(M)=G I S(N)=0$ and $S(M)=$ $S(N)=\frac{-c_{1}-d_{1}}{2}, H(M)=H(N)=\frac{c_{1}+d_{1}}{2}$. This implies that $M=N$. This example shows that none of these methods $[1,2,6]$ are better to each other;

9. Comparison of GIS score function with Novel accuracy score function $M$ in Ye [2]: Using example 2.1. in [6], Garg has shown the inconsistency of Ye's [2] method. However, his method also fails to compare arbitrary IVIFNs which we can be see from the following example.

Let $A=\left([0,0],\left[c_{1}, d_{1}\right]\right)$ and $B=\left([0,0],\left[c_{2}, d_{2}\right]\right)=\left([0,0],\left[c_{1}-\epsilon, d_{1}+\epsilon\right]\right), \forall \epsilon \in$ $[0,1], \epsilon<c_{1}$ be any two IVIFNs. If we apply Ye's [2] method, then we get $M(A)=$ $M(B)=\frac{c_{1}+d_{1}-2}{2} \Rightarrow A=B$. If we apply GIS to $A$ and $B$, then we get $A=B$. However, $A$ and $B$ are different. Hence, both of these methods are not better to each other in ranking arbitrary IVIFNs; 
10. Comparison of GIS function with accuracy score function $L$ in Nayagam et al. [7]: Using Example 2.1. in [6], Garg has shown the inconsistency of Nayagam et al.'s [7] method. The following example shows the illogical result of GIS and $L$ score functions. Let $A=\left([0,0],\left[c_{1}, d_{1}\right]\right)$ and $B=\left([0,0],\left[c_{2}, d_{2}\right]\right)=\left([0,0],\left[c_{1}-\epsilon, d_{1}+\right.\right.$ $\epsilon]), \forall \epsilon \in[0,1], \epsilon<c_{1}$ be any two IVIFNs. If we apply Garg's and Nayagam et al.'s [7] method to $A$ and $B$, then we get $\operatorname{GIS}(A)=\operatorname{GIS}(B)=0$ and $L(A)=L(B)=\frac{-\left(c_{1}+d_{1}\right)}{2}$, respectively. This implies that $A=B$. Hence, both the methods are illogical in comparing arbitrary IVIFNs of the above type.

11. Comparison of GIS function with an improved score function $I$ in Bai [5]: Since Garg's [6] method is the generalization of Bai's [5] improved score function, Garg's method also has the same drawbacks of Bai's [5] improved score function. The following example shows the illogicality of both the methods. Let $M=\left([0,0],\left[c_{1}, d_{1}\right]\right)$ and $N=\left([0,0],\left[c_{2}, d_{2}\right]\right)$ be any two IVIFNs. Then we get $\operatorname{GIS}(M)=G I S(N)=0$ and $I(M)=I(N)=0$. Hence, both the methods are not logical in ranking IVIFNs.

Hence, from the above examples, we may conclude that none of the familiar methods $[1,2,5-7]$ can rank arbitrary IVIFNs, which makes the pathway for defining a new score function on the class of IVIFNs for comparing any two IVIFNs and also GIS did not define a total order on the set of IVIFNs, which is seen from the incorrectness of Theorem 3.1 (in page no. 5 of [6]). In 2016, Nayagam et al. [10] have defined a non-hesitance score for ranking arbitrary IVIFNs. The ranking principle that was introduced by Nayagam et al. [10] has overcome all the drawbacks mentioned above.

\section{Comparison with Nayagam et al.'s Ranking Principle}

In this subsection, first we present a short review of Nayagam et al.'s [10] ranking approach for comparing arbitrary IVIFNs and then the illogicality/limitations of different ranking approaches introduced in [1,2,5-7] is shown by illustrative examples. Finally the advantage of Nayagam et al.'s [10] ranking principle is shown by comparing it with the approaches in $[1,2,5-7]$.

Definition 9. (Nayagam et al., 2016) Let $A=([a, b],[c, d])$ be an IVIFN. Then, the non-hesitance score function of $A$ is defined as $J(A)=\frac{a+b+c-d+a b+c d}{3}$.

The efficiency of Nayagam et al.'s [10] approach in overcoming the Limitations $I_{1}$ to $I_{7}$ and the drawbacks 1-5 are shown in the following table.

Hence, from Table 1, it is apparent that the shortcomings of all the ranking methods introduced in [1,2,5-7] are overcome by Nayagam et al.'s [10] ranking principle. However, Nayagam et al.'s [10] non-hesitance score function is also failed to rank arbitrary IVIFNs in some places, which can be seen from the following example.

Table 1. Comparison of some existing score functions with Nayagam et al.'s [10] approach.

\begin{tabular}{|c|c|c|}
\hline Numerical Example & Shortcomings of Existing Methods & Nayagam et al.'s Ranking Principle \\
\hline $\begin{array}{l}\overline{A_{1}}=([0,0][0.4,0.5]) \\
A_{2}=([0,0][0.5,0.7])\end{array}$ & $\operatorname{GIS}\left(A_{1}\right)=\operatorname{GIS}\left(A_{2}\right) \Rightarrow A_{1}=A_{2}$ & $J\left(A_{1}\right)=0.033<J\left(A_{2}\right)=0.05 \Rightarrow A_{1}<A_{2}$ \\
\hline $\begin{array}{l}A_{1}=([0,0][0.5,0.6]) \\
A_{2}=([0,0][0.55,0.55])\end{array}$ & $\begin{array}{l}\operatorname{GIS}\left(A_{1}\right)=0=\operatorname{GIS}\left(A_{2}\right) \Rightarrow A_{1}=A_{2} \\
S\left(A_{1}\right)=S\left(A_{2}\right)=-0.55, H\left(A_{1}\right)=H\left(A_{2}\right)= \\
0.55 \Rightarrow A_{1}=A_{2}\end{array}$ & $\begin{array}{l}J\left(A_{1}\right)=0.067<J\left(A_{2}\right)=0.101 \Rightarrow A_{1}<A_{2} \\
M\left(A_{1}\right)=M\left(A_{2}\right)=-0.45 \Rightarrow A_{1}=A_{2}\end{array}$ \\
\hline $\begin{array}{l}A=([0,0],[0.2,0.4]) \\
B=([0,0],[0.3,0.5])\end{array}$ & $\operatorname{GIS}(A)=0=\operatorname{GIS}(B) \Rightarrow A=B$ & $J(A)=-0.04<J(B)=-0.017 \Rightarrow A<B$ \\
\hline $\begin{array}{l}A_{1}=([0,0],[0.1,0.5]) \\
A_{2}=([0,0],[0.3,0.7])\end{array}$ & $\begin{array}{l}\operatorname{GIS}\left(A_{1}\right)=0=\operatorname{GIS}\left(A_{2}\right) \Rightarrow A_{1}=A_{2} \\
I\left(A_{1}\right)=I\left(A_{2}\right)=0 \Rightarrow A_{1}=A_{2}\end{array}$ & $J\left(A_{1}\right)=-0.117<J\left(A_{2}\right)=-0.063 \Rightarrow A_{1}<A_{2}$ \\
\hline
\end{tabular}


Example 1. Let $A=\langle[0.1,0.4],[0.1,0.2]\rangle, B=\langle[0.2,0.25],[0.2,0.3]\rangle \in \operatorname{IVIFN}$. Now $J(A)=$ 0.15333 and $J(B)=0.15333 \Rightarrow A=B$. But $A \neq B$.

Hence, there is a need for another parameter for ranking IVIFNs. In the following section, a new score function has been introduced, which measures the non-membershipness of an IVIFN for ranking arbitrary IVIFNs.

\section{A New Non-Membership Score of IVIFNs}

This section proposes a non-membership score function for ranking arbitrary IVIFNs and examines some of its properties using illustrative examples.

Definition 10. Let $A=\left(\left[a_{1}, b_{1}\right],\left[c_{1}, d_{1}\right]\right), B=\left(\left[a_{2}, b_{2}\right],\left[c_{2}, d_{2}\right]\right) \in \operatorname{IVIFN.} A \subseteq_{2} B$ if $a_{1} \geq$ $a_{2}, b_{1} \leq b_{2}, c_{1} \geq c_{2}$ and $d_{1} \geq d_{2}$.

Definition 11. Let $A=\left\langle\left[a_{1}, b_{1}\right],\left[c_{1}, d_{1}\right]\right\rangle \in I V I F N$. Then the non-membership score function for $A$ is defined as $N_{M}(A)=\frac{a_{1}-b_{1}+c_{1}+d_{1}+a_{1} b_{1}+c_{1} d_{1}}{3}$.

Proofs of the following propositions are immediate from the Definition 11. Hence, they are omitted.

Proposition 1. For any real number $r \in[0,1 / 2], N_{M}(r)=\frac{2 r(1+r)}{3}$.

Proposition 2. If $A=\left(a_{1}, 1-a_{1}\right)$ is a fuzzy number, then $N_{M}(A)=1-\frac{2 a_{1}\left(2-a_{1}\right)}{3}$.

Proposition 3. If $A=\left(a_{1}, c_{1}\right)$ is an intuitionistic fuzzy number, then $N_{M}(A)=\frac{a_{1}^{2}+2 c_{1}+c_{1}^{2}}{3}$.

Proposition 4. Let $A=\left(\left[a_{1}, b_{1}\right],\left[1-b_{1}, 1-a_{1}\right]\right)$ be an interval-valued fuzzy number then $N_{M}(A)=\frac{3\left(1-b_{1}\right)-a_{1}\left(1-2 b_{1}\right)}{3}$.

Definition 12. Let $A, B \in I V I F N$. If $N_{M}(A) \geq N_{M}(B)$ then $A \leq B$.

Theorem 1. Let $A, B \in I V I F N$. If $A \subseteq_{2} B$, then $N_{M}(A) \geq N_{M}(B)$.

Proof. Let $A, B \in I V I F N$. Assume: $A \subseteq_{2} B \Rightarrow a_{1} \geq a_{2}, b_{1} \leq b_{2}, c_{1} \geq c_{2}, d_{1} \geq d_{2} \ldots$ (1)

We claim that $N_{M}(A) \geq N_{M}(B) \Rightarrow N_{M}(A)-N_{M}(B) \geq 0$.

$2\left(N_{M}(A)-N_{M}(B)\right)=\left(a_{1}-b_{1}+c_{1}+d_{1}+a_{1} b_{1}+c_{1} d_{1}\right)-\left(a_{2}-b_{2}+c_{2}+d_{2}+a_{2} b_{2}+\right.$ $\left.c_{2} d_{2}\right)=\left(a_{1}-a_{2}\right)+\left(b_{2}-b_{1}\right)+\left(c_{1}-c_{2}\right)+\left(d_{1}-d_{2}\right)+\left(a_{1} b_{1}-a_{2} b_{2}\right)+\left(c_{1} d_{1}-c_{2} d_{2}\right) \ldots$ (2) Add and subtract $a_{1} b_{2}$ from (2), we get, $2\left(N_{M}(A)-N_{M}(B)\right)=\left(a_{1}-a_{2}\right)+\left(b_{2}-\right.$ $\left.b_{1}\right)+\left(c_{1}-c_{2}\right)+\left(d_{1}-d_{2}\right)+\left(a_{1} b_{1}-a_{1} b_{2}+a_{1} b_{2}-a_{2} b_{2}\right)+\left(c_{1} d_{1}-c_{2} d_{2}\right) \Rightarrow 2\left(N_{M}(A)-\right.$ $\left.N_{M}(B)\right)=\left(a_{1}-a_{2}\right)\left(1+b_{2}\right)+\left(b_{2}-b_{1}\right)\left(1-a_{1}\right)+\left(c_{1}-c_{2}\right)+\left(d_{1}-d_{2}\right)+\left(c_{1} d_{1}-c_{2} d_{2}\right) \geq 0$, from (1).

Hence, $\left(N_{M}(A)-N_{M}(B)\right) \geq 0$. Hence the proof.

Theorem 2. Let $A, B, C \in I V I F N$ and $A \subseteq_{2}$ B. If $N_{M}(A) \geq N_{M}(B)$, then $N_{M}(A+C) \geq$ $N_{M}(B+C)$.

Proof. Let $A=\left(\left[a_{1}, b_{1}\right],\left[c_{1}, d_{1}\right]\right), B=\left(\left[a_{2}, b_{2}\right],\left[c_{2}, d_{2}\right]\right)$ and $C=([a, b],[c, d])$ be any three IVIFNs. $A \subseteq_{2} B \Rightarrow a_{1} \geq a_{2}, b_{1} \leq b_{2}, c_{1} \geq c_{2}, d_{1} \geq d_{2}$. . . (3)

Using Definition 8 , we get, $A+C=\left(\left[a_{1}+a-a a_{1}, b_{1}+b-b b_{1}\right],\left[c c_{1}, d d_{1}\right]\right)$ and $B+$ $C=\left(\left[a_{2}+a-a a_{2}, b_{2}+b-b b_{2}\right],\left[c c_{2}, d d_{2}\right]\right)$.

It is very clear that, $a_{1}+a-a a_{1} \geq a_{2}+a-a a_{2}, b_{1}+b-b b_{1} \leq b_{2}+b-b b_{2}, c c_{1} \geq$ $c c_{2}, d d_{1} \geq d d_{2} \Rightarrow A+C \subseteq_{2} B+C$.

Therefore, from Theorem $1, N_{M}(A+C) \geq N_{M}(B+C)$. Hence the proof. 
Example 2. Let $A=\langle[0.1,0.4],[0.1,0.2]\rangle, B=\langle[0.2,0.25],[0.2,0.3]\rangle \in \operatorname{IVIFN}$. Now $J(A)=$ 0.15333 and $J(B)=0.15333 \Rightarrow A=B$. However, $A$ and $B$ are different IVIFNs. If we apply $N_{M}$ to $A$ and $B$, then we get $N_{M}(A)=0.02<N_{M}(B)=0.186667$. Hence $A>B$ which favors human intuition.

Example 3. Let $A=\langle[0.4,0.6],[0,0.15]\rangle, B=\langle[0.45,0.55],[0,0.15]\rangle \in I V I F N$. If we apply our new non-membership score to $A$ and $B$, then we get $N_{M}(A)=0.063, N_{M}(B)=0.099167$ and $N_{M}(B)>N_{M}(A)$. This implies that $A>B$.

Example 4. Let $A=\langle[0.2,0.3],[0.7,0.7]\rangle, B=\langle[0.3,0.3],[0.6,0.6]\rangle \in I V I F N$. If we apply our new non-membership score to $A$ and $B$, then we get $N_{M}(A)=0.6167$ and $N_{M}(B)=0.55 \Rightarrow$ $A<B$.

The efficacy of the proposed non-membership based score function in overcoming the Limitations $I_{1}$ to $I_{6}$ are shown in Table 2.

Table 2. Comparison of some existing score function with the proposed non-membership based score function.

\begin{tabular}{|c|c|c|}
\hline Numerical Example & Shortcomings of Existing Methods & Proposed Ranking Principle \\
\hline $\begin{array}{l}A_{1}=([0,0][0.1,0.3]) \\
A_{2}=([0,0][0.7,0.9])\end{array}$ & $\operatorname{GIS}\left(A_{1}\right)=\operatorname{GIS}\left(A_{2}\right) \Rightarrow A_{1}=A_{2}$ & $\begin{array}{l}N_{M}\left(A_{1}\right)=0.143<N_{M}\left(A_{2}\right)=0.743 \Rightarrow A_{1}>A_{2} \\
\text { which favors with human intuition. }\end{array}$ \\
\hline $\begin{array}{l}A_{1}=([0,0][0.6,0.7]) \\
A_{2}=([0,0][0.65,0.65])\end{array}$ & $\begin{array}{l}\operatorname{GIS}\left(A_{1}\right)=0=\operatorname{GIS}\left(A_{2}\right) \Rightarrow A_{1}=A_{2} \\
S\left(A_{1}\right)=S\left(A_{2}\right)=-0.65, H\left(A_{1}\right)= \\
H\left(A_{2}\right)=0.65 \Rightarrow A_{1}=A_{2}\end{array}$ & $\begin{array}{l}N_{M}\left(A_{1}\right)=0.573<N_{M}\left(A_{2}\right)=0.574 \Rightarrow A_{1}>A_{2} \\
M\left(A_{1}\right)=M\left(A_{2}\right)=-0.35 \Rightarrow A_{1}=A_{2}\end{array}$ \\
\hline $\begin{array}{l}A_{1}=([0,0],[0.2,0.3]) \\
A_{2}=([0,0],[0.4,0.5])\end{array}$ & $\operatorname{GIS}\left(A_{1}\right)=0=\operatorname{GIS}\left(A_{2}\right) \Rightarrow A_{1}=A_{2}$ & $N_{M}\left(A_{1}\right)=0.19<N_{M}\left(A_{2}\right)=0.37 \Rightarrow A_{1}>A_{2}$ \\
\hline $\begin{array}{l}A=([0.3,0.7],[0.3,0.3]) \\
B=([0.4,0.7],[0.1,0.2])\end{array}$ & $J(A)=J(B)=0.433 \Rightarrow A=B$ & $N_{M}(A)=0.167>N_{M}(B)=0.1 \Rightarrow A<B$ \\
\hline $\begin{array}{l}A=([0.2,0.3],[0.7,0.7]) \\
B=([0.3,0.3],[0.6,0.6])\end{array}$ & $J(A)=J(B)=0.35 \Rightarrow A=B$ & $N_{M}(A)=0.617>N_{M}(B)=0.55 \Rightarrow A<B$ \\
\hline
\end{tabular}

Hence, from Table 2, it is very clear that the shortcomings of all the ranking methods introduced in $[1,2,5-7,10]$ can be eliminated by the proposed ranking principle.

\section{Interval-Valued Intuitionistic Fuzzy TOPSIS}

This section presents a new algorithm for solving MCDM problems using the IVIFTOPSIS method. TOPSIS is one of the most popular MCDM methods introduced by Hwang and Yoon [15]. Any IVIF-MCDM problem is mathematically defined as follows.

Let $M=\left\{M_{1}, M_{2}, \ldots, M_{r}\right\}$ be the set of $r$ alternatives and let $C=\left\{C_{1}, C_{2}, \ldots, C_{s}\right\}$ be the set of $s$ criteria based on which the alternatives to be evaluated. Let $N=\left(n_{i j}\right)_{r \times s}$ be the decision matrix, where $n_{i j}$ represents the performance of $M_{i}(i$-th alternative ) with respect to the $C_{j}$ ( $j$-th criterion). In this paper, every $n_{i j}$ is represented by an IVIFN, that is, $n_{i j}=\left(\left[a_{i j}, b_{i j}\right],\left[c_{i j}, d_{i j}\right]\right)$, where $n_{i j}$ represents the membership and nonmembership degree of alternative $M_{i}$ with respect to criterion $C_{j}$.

Algorithm 1 for solving the IVIF-MCDM problem is given as follows: 


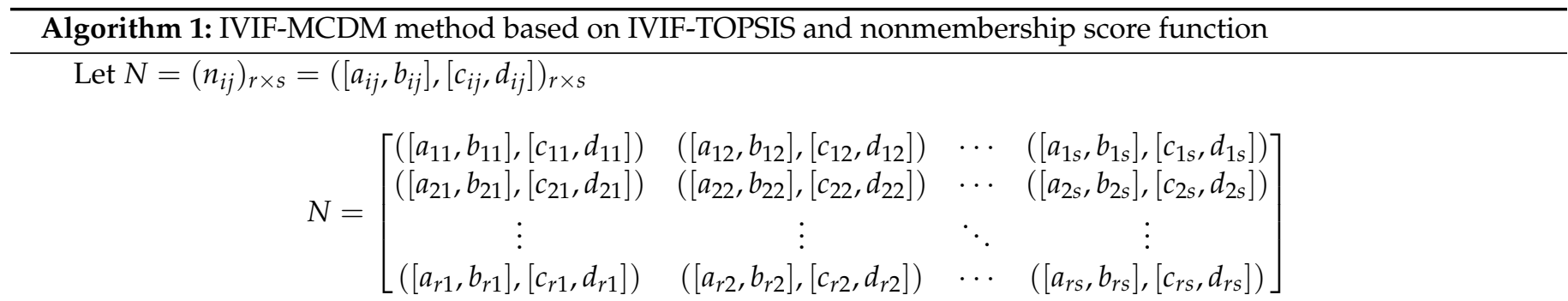

be the decision matrix.

1. Score Matrix $\left(N^{\prime}\right)$ :

A new $r \times s$ score matrix $N^{\prime}$ is formed by applying non-membership score function to the each entry of the decisionmatrix $N$. i.e., $N^{\prime}=\left(s_{i j}\right)_{r \times s}$, where $s_{i j}=N_{M}\left(n_{i j}\right)$;

2. Interval-valued intuitionistic fuzzy positive ideal solution (IVIFPIS):

An interval-valued intuitionistic fuzzy positive ideal solution (IVIFPIS) denoted by $P_{I S}$, is the set of IVIFNs, where the number of IVIFNs in $P_{I S}$ is equal to $s$ (the number of columns in the decision matrix); that is, $P_{I S}=\left\{P_{1}, P_{2}, \ldots, P_{s}\right\}$. Each $P_{i}$ is an IVIFN and is defined as:

$P_{1}=\left(\left[\max \left(a_{11}, a_{21}, \ldots, a_{r 1}\right), \max \left(b_{11}, b_{21}, \ldots, b_{r 1}\right)\right],\left[\min \left(c_{11}, c_{21}, \ldots, c_{r 1}\right), \min \left(d_{11}, d_{21}, \ldots, d_{r 1}\right)\right]\right)$

$P_{2}=\left(\left[\max \left(a_{12}, a_{22}, \ldots, a_{r 2}\right), \max \left(b_{12}, b_{22}, \ldots, b_{r 2}\right)\right],\left[\min \left(c_{12}, c_{22}, \ldots, c_{r 2}\right), \min \left(d_{12}, d_{22}, \ldots, d_{r 2}\right)\right]\right)$ :

$P_{s}=\left(\left[\max \left(a_{1 s}, a_{2 s}, \ldots, a_{r s}\right), \max \left(b_{1 s}, b_{2 s}, \ldots, b_{r s}\right)\right],\left[\min \left(c_{1 s}, c_{2 s}, \ldots, c_{r s}\right), \min \left(d_{1 s}, d_{2 s}, \ldots, d_{r s}\right)\right]\right) ;$

3. Interval-valued intuitionistic fuzzy negative ideal solution (IVIFNIS):

An interval-valued intuitionistic fuzzy negative ideal solution (IVIFNIS) denoted by $N_{I S}$, is the set of IVIFNs where the number of IVIFNs in $N_{I S}$ is equal to $s$ (the number of criteria available in the problem), that is,

$N_{I S}=\left\{N_{1}, N_{2}, \ldots, N_{s}\right\}$. Each $N_{i}$ is an IVIFN and is defined as:

$N_{1}=\left(\left[\min \left(a_{11}, a_{21}, \ldots, a_{r 1}\right), \min \left(b_{11}, b_{21}, \ldots, b_{r 1}\right)\right],\left[\max \left(c_{11}, c_{21}, \ldots, c_{r 1}\right), \max \left(d_{11}, d_{21}, \ldots, d_{r 1}\right)\right]\right)$

$N_{2}=\left(\left[\min \left(a_{12}, a_{22}, \ldots, a_{r 2}\right), \min \left(b_{12}, b_{22}, \ldots, b_{r 2}\right)\right],\left[\max \left(c_{12}, c_{22}, \ldots, c_{r 2}\right), \max \left(d_{12}, d_{22}, \ldots, d_{r 2}\right)\right]\right)$

$\vdots$

$N_{s}=\left(\left[\min \left(a_{1 s}, a_{2 s}, \ldots, a_{r s}\right), \min \left(b_{1 s}, b_{2 s}, \ldots, b_{r s}\right)\right],\left[\max \left(c_{1 s}, c_{2 s}, \ldots, c_{r s}\right), \max \left(d_{1 s}, d_{2 s}, \ldots, d_{r s}\right)\right]\right)$

4. Non-membership score function of IVIFPIS and IVIFNIS:

Non-membership score functions for IVIFPIS and IVIFNIS are denoted by $N_{M}\left(P_{I S}\right)$,

$N_{M}\left(N_{I S}\right)$ and are defined as:

$N_{M}\left(P_{I S}\right)=\left\{N_{M}\left(P_{1}\right), N_{M}\left(P_{2}\right), \ldots, N_{M}\left(P_{S}\right)\right\}$ and $N_{M}\left(N_{I S}\right)=\left\{N_{M}\left(N_{1}\right), N_{M}\left(N_{2}\right), \ldots, N_{M}\left(N_{s}\right)\right\} ;$

5. Distance between aggregated performance of alternatives and IVIFPIS, IVIFNIS:

The aggregated performance of an alternatives, non-membership score functions of IVIFPIS and IVIFNIS are obatined as real numbers which can be seen from Step 1 and Step 4 . Hence, the distance

between them can be calculated using the following formula:

$d_{i}^{*}\left(N_{M}\left(M_{i}\right), N_{M}\left(P_{I S}\right)\right)=\sum_{j=1}^{s}\left(w_{j}\left|N_{M}\left(n_{i j}\right)-N_{M}\left(P_{j}\right)\right|\right)$,

$d_{i}^{-}\left(N_{M}\left(M_{i}\right), N_{M}\left(N_{I S}\right)\right)=\sum_{j=1}^{s}\left(w_{j}\left|N_{M}\left(n_{i j}\right)-N_{M}\left(N_{j}\right)\right|\right)$,

where $d_{i}^{*}\left(N_{M}\left(M_{i}\right), N_{M}\left(P_{I S}\right)\right), d_{i}^{-}\left(N_{M}\left(M_{i}\right), N_{M}\left(N_{I S}\right)\right)$ represent the distance between alternative $M_{i}$ and IVIFPIS, IVIFNIS, respectively, and $w_{j}$ is the weight of the $j$-th criterion;

6. Closeness Coefficient of Alternative $\left(C_{i}\left(M_{i}\right)\right)$ :

Relative closeness of an alternative $M_{i}$ is defined as,

$C_{i}\left(M_{i}\right)=\frac{d_{i}^{-}\left(N_{M}\left(M_{i}\right), N_{M}\left(N_{I S}\right)\right)}{d_{i}^{*}\left(N_{M}\left(M_{i}\right), N_{M}\left(P_{I S}\right)\right)+d_{i}^{-}\left(N_{M}\left(M_{i}\right), N_{M}\left(N_{I S}\right)\right)}$. The values of the closeness coefficient decides the Ranking of alternatives $\left(M_{i}\right)$. The alternative with the highest closeness coefficient will be ranked first.

\section{Numerical Illustrations}

This subsection demonstrates the applicability of the proposed algorithm in solving the IVIF-MCDM problem using numerical examples.

Example 5. An animation expert wants to select the best computer animation work for an award from four possible alternatives $\left(A_{1}, A_{2}, A_{3}, A_{4}\right)$. The expert has to make a decision according to 
the following three criteria, (a) $C_{1}$ is the artistic appeal, (b) $C_{2}$ is the visual effect and (c) $C_{3}$ is the creative script. The criteria weight is given by $W=\{0.35,0.25,0.40\}$.

Four alternatives are evaluated by utilizing interval-valued intuitionistic fuzzy numbers given by the decision maker under three different criteria. The performance of an alternative under three criteria is obtained from the decision maker and given in the following matrix:

$$
N=\left(n_{i j}\right)_{4 \times 3}=\left[\begin{array}{lll}
([0.4,0.5],[0.3,0.4]) & ([0.4,0.6],[0.2,0.4]) & ([0.1,0.3],[0.5,0.6]) \\
([0.6,0.7],[0.2,0.3]) & ([0.6,0.7],[0.2,0.3]) & ([0.4,0.7],[0.1,0.2]) \\
([0.3,0.6],[0.3,0.4]) & ([0.5,0.6],[0.3,0.4]) & ([0.5,0.6],[0.1,0.3]) \\
([0.7,0.8],[0.1,0.2]) & ([0.6,0.7],[0.1,0.3]) & ([0.3,0.4],[0.1,0.2])
\end{array}\right]
$$

- Score matrix of a given decision matrix $N$ is obtained using step 1 of Algorithm 1 and given below:

$$
S=\left(s_{i j}\right)_{4 \times 3}=\left[\begin{array}{lll}
0.307 & 0.240 & 0.410 \\
0.293 & 0.293 & 0.100 \\
0.233 & 0.340 & 0.210 \\
0.260 & 0.250 & 0.113
\end{array}\right]
$$

- Interval-valued intuitionistic fuzzy positive ideal solution (IVIFPIS) and negative ideal solution for given problem is obtained using step 2 and step 3 of Algorithm 1, that is, $P_{I S}=\left\{P_{1}, P_{2}, P_{3}\right\}=\{([0.7,0.8],[0.1,0.2]),([0.6,0.7],[0.1,0.3]),([0.5,0.7],[0.1,0.2])\}$, $N_{I S}=\left\{N_{1}, N_{2}, N_{3}\right\}=\{([0.3,0.5],[0.3,0.4]),([0.4,0.6],[0.3,0.4]),([0.1,0.3],[0.5,0.6])\}$.

- $\quad$ Non-membership scores of IVIFPIS and IVIFNIS are calculated using step 4 of Algorithm 1. $N_{M}\left(P_{I S}\right)=\{0.26,0.25,0.157\}, N_{M}\left(N_{I S}\right)=\{0.257,0.287,0.41\} ;$

- $\quad$ Distance between performance of alternative and IVIFPIS, IVIFNIS is obtained using step 5 .

$d_{1}^{*}\left(A_{1}, P_{1}\right)=0.120, d_{2}^{*}\left(A_{2}, P_{2}\right)=0.045, d_{3}^{*}\left(A_{3}, P_{3}\right)=0.053, d_{4}^{*}\left(A_{4}, P_{4}\right)=0.017$ and $d_{1}^{-}\left(A_{1}, N_{1}\right)=0.029, d_{2}^{-}\left(A_{2}, N_{2}\right)=0.139, d_{3}^{-}\left(A_{3}, N_{3}\right)=0.102, d_{4}^{-}\left(A_{4}, N_{4}\right)=0.129 ;$

- Closeness coefficient of four alternatives are obtained using step 6 of Algorithm 1 , that is, $C_{1}\left(A_{1}\right)=0.195, C_{2}\left(A_{2}\right)=0.754, C_{3}\left(A_{3}\right)=0.656, C_{4}\left(A_{4}\right)=0.881$, which ranks the alternative $A_{4}$ as the best among four alternatives and the ranking order is $A_{4}>A_{2}>A_{3}>A_{1}$, which favors the human intuition.

Example 6. A committee wants to choose the best candidate for one faculty position available in the Department of Mathematics. Six candidates $\left(A_{1}, A_{2}, \ldots, A_{6}\right)$ have been called for interview and their performances have been evaluated based on the following six criteria: (1) $\mathrm{Cr}_{1}$ Work attitude, (2) $\mathrm{Cr}_{2}$ Teaching methodology, (3) $\mathrm{Cr}_{3}$ Previous Research Output, (4) $\mathrm{Cr}_{4}$ Exploration Capacity, (5) $C r_{5}$ Future Research Plan, (6) $C r_{6}$ Experience. The weights $\left(w_{i}\right)$ for attributes $C r_{i}, i=1,2 \ldots, 6$ are assumed as follows: $W=\left\{w_{1}, w_{2}, \ldots, w_{6}\right\}=\{0.15,0.2,0.15,0.1,0.2,0.2\}$.

Six candidates are evaluated based on their interview performance. Performance of six candidates under six criteria are represented by IVIFNs and which are given in decision matrix $M=\left(m_{i j}\right)_{6 \times 6}$, where $m_{i j}$ represents the performance of candidate $A_{i}$ with respect to criteria $C r_{j}, i, j=1,2, \ldots, 6$.

$$
M=\left[\begin{array}{cccc}
C r_{1} & C r_{2} & C r_{3} \\
A_{1} & ([0.2,0.3],[0.4,0.5]) & ([0.6,0.7],[0.2,0.3]) & ([0.4,0.5],[0.3,0.4]) \\
A_{2} & ([0.5,0.5],[0.1,0.2]) & ([0.6,0.7],[0.1,0.2]) & ([0.4,0.5],[0.3,0.4]) \\
A_{3} & ([0.4,0.5],[0.2,0.3]) & ([0.5,0.5],[0.2,0.3]) & ([0.3,0.4],[0.1,0.2]) \\
A_{4} & ([0.1,0.3],[0.2,0.4]) & ([0.5,0.7],[0.1,0.2]) & ([0.1,0.2] .[0.5,0.7]) \\
A_{5} & ([0.2,0.4],[0.1,0.4]) & ([0.1,0.3],[0.3,0.6]) & ([0.2,0.4],[0.2,0.4]) \\
A_{6} & ([0.4,0.5],[0.1,0.2]) & ([0.5,0.6],[0,0.2]) & ([0.4,0.5],[0.3,0.5])
\end{array}\right]
$$




$$
\left[\begin{array}{cccc} 
& C r_{4} & C r_{5} & C r_{6} \\
A_{1} & ([0.6,0.7],[0.1,0.2]) & ([0.3,0.4],[0.1,0.2]) & ([0.5,0.6],[0.3,0.4]) \\
A_{2} & ([0.6,0.7],[0,0.1]) & ([0.4,0.5],[0.1,0.2]) & ([0.6,0.7],[0.2,0.2]) \\
A_{3} & ([0.1,0.2],[0.4,0.5]) & ([0.3,0.5],[0.3,0.4]) & ([0.3,0.4],[0.1,0.4]) \\
A_{4} & ([0.1,0.1],[0.6,0.7]) & ([0.2,0.3],[0.3,0.5]) & ([0.1,0.3],[0.2,0.4]) \\
A_{5} & ([0.1,0.2],[0.2,0.3]) & ([0.2,0.3],[0.1,0.3]) & ([0.3,0.4],[0.4,0.4]) \\
A_{6} & ([0.5,0.6],[0.1,0.2]) & ([0.3,0.4],[0,0.2]) & ([0.55,0.6],[0.1,0.2])
\end{array}\right]
$$

Algorithm 1 is used to solve the selection problem.

- $\quad$ Score matrix $S=\left(s_{i j}\right)_{6 \times 6}$ for given $M$ is obtained using step 1 .

$$
S=\left[\begin{array}{ccccccc} 
& C r_{1} & C r_{2} & C r_{3} & C r_{4} & C r_{5} & C r_{6} \\
A_{1} & 0.353 & 0.293 & 0.307 & 0.213 & 0.113 & 0.34 \\
A_{2} & 0.19 & 0.213 & 0.307 & 0.14 & 0.14 & 0.253 \\
A_{3} & 0.22 & 0.27 & 0.113 & 0.34 & 0.257 & 0.187 \\
A_{4} & 0.17 & 0.157 & 0.49 & 0.577 & 0.303 & 0.17 \\
A_{5} & 0.14 & 0.303 & 0.187 & 0.16 & 0.13 & 0.327 \\
A_{6} & 0.14 & 0.133 & 0.35 & 0.173 & 0.073 & 0.2
\end{array}\right]
$$

- Interval-valued intuitionistic fuzzy positive and negative ideal solutions IVIFPIS and IVIFNIS are obtained using step 2 and step 3 of Algorithm 1.

$P_{I S}=\{([0.5,0.5][0.1,0.2]),([0.6,0.7],[0,0.2]),([0.4,0.5],[0.1,0.2]),([0.6,0.7],[0,0.1])$, $([0.4,0.5],[0,0.2]),([0.6,0.7],[0.1,0.2])\}, \quad N_{I S}=\{([0.1,0.3],[0.4,0.5])$, $([0.1,0.3],[0.3,0.6]),([0.1,0.2],[0.5,0.7]),([0.1,0.1],[0.6,0.7]),([0.2,0.3],[0.3,0.5])$, $([0.1,0.3],[0.4,0.4])\}$.

- Non-membership scores for IVIFPIS and IVIFNIS are calculated using the proposed score function and step 4 of Algorithm 1.

$N_{M}\left(P_{I S}\right)=\{0.19,0.173,0.14,0.14,0.1,0.213\}, N_{M}\left(N_{I S}\right)=\{0.31,0.303,0.49,0.577$, $0.303,0.263\}$.

- Distance between alternative $A_{i}$ and IVIFPIS, IVIFNIS are calculated using step 5 and given as follows:

$d_{1}^{*}\left(A_{1}, P_{1}\right)=0.109, d_{2}^{*}\left(A_{2}, P_{2}\right)=0.049, d_{3}^{*}\left(A_{3}, P_{3}\right)=0.085, d_{4}^{*}\left(A_{4}, P_{4}\right)=0.152$, $d_{5}^{*}\left(A_{5}, P_{5}\right)=0.071, d_{6}^{*}\left(A_{6}, P_{6}\right)=0.058$ and $d_{1}^{-}\left(A_{1}, N_{1}\right)=0.126, d_{2}^{-}\left(A_{2}, N_{2}\right)=0.142$, $d_{3}^{-}\left(A_{3}, N_{3}\right)=0.125, d_{4}^{-}\left(A_{4}, N_{4}\right)=0.069, d_{5}^{-}\left(A_{5}, N_{5}\right)=0.160, d_{6}^{-}\left(A_{6}, N_{6}\right)=0.180 ;$

- Closeness coefficient of six alternatives are obtained using step 6 of Algorithm 1, that is, $C_{1}\left(A_{1}\right)=0.536, C_{2}\left(A_{2}\right)=0.743, C_{3}\left(A_{3}\right)=0.597, C_{4}\left(A_{4}\right)=0.313$, $C_{5}\left(A_{5}\right)=0.692, C_{6}\left(A_{6}\right)=0.755$ and the ranking is $A_{6}>A_{2}>A_{5}>A_{3}>A_{1}>A_{4}$. Hence, candidate $6\left(A_{6}\right)$ should be selected for the faculty position.

\section{Conclusions and Future Scope}

A new non-membership score function has been introduced for the effective comparison of any two arbitrary IVIFNs. Besides, the effectiveness of the proposed nonmembership score function has been demonstrated by comparing it with different existing methods. Finally, the application of the proposed non-membership score function in solving IVIF-MCDM problems using the IVIF-TOPSIS method has been demonstrated through numerical examples. The proposed score function defines a total ordering on the particular subset of IVIFNs and does not define a total order on the entire class of IVIFNs. Therefore, a total ordering principle on the class of IVIFNs would be aimed in further research. Similar to IVIFNs, interval-valued Pythagorean fuzzy numbers (IVPFNs) [16], interval-valued Fermatean fuzzy numbers (IVFFNs) [17], and so forth, are also used in the literature for modelling real-life problems with incomplete information. Hence, the proposed non-membership score function can be extended to the class of IVPFNs and IVFFNs. Using this, a new MCDM algorithm can be developed for solving problems modelled under the IVPFN and IVFFN environment. Researchers can also introduce a 
new similarity on the set of IVPFNs and IVFFNs by using the idea of the non-membership score function.

Author Contributions: Conceptualization, J.S.; methodology, J.S. and A.M.; formal analysis, J.S. and A.M.; investigation, J.S.; resources, J.S. and A.M.; writing-original draft preparation, J.S.; writingreview and editing, J.S. and A.M.; funding acquisition, J.S. All authors have read and agreed to the published version of the manuscript.

Funding: Corresponding Author thanks Atal Bihari Vajpayee Indian Institute of Information Technology and Management Gwalior for funding this research.

Institutional Review Board Statement: Not applicable.

Informed Consent Statement: Not applicable.

Data Availability Statement: Not applicable.

Acknowledgments: Authors thank the Editor, Associate Editor and the anonymous reviewers for their valuable suggestions.

Conflicts of Interest: The authors declare no conflict of interest.

\section{References}

1. $\mathrm{Xu}, \mathrm{Z} . \mathrm{S}$. Methods for aggregating interval-valued intuitionistic fuzzy information and their application to decision making. Control Decis. 2007, 22, 215-219.

2. Ye, J. Multicriteria fuzzy decision-making method based on a novel accuracy function under interval-valued intuitionistic fuzzy environment. Expert Syst. Appl. 2009, 36, 6899-6902. [CrossRef]

3. Nayagam, V.L.G.; Geetha, S. Ranking of interval-valued intuitionistic fuzzy sets. Appl. Soft Comput. 2011, 11, 3368-3372. [CrossRef]

4. Geetha, S.; Nayagam, V.L.G.; Ponalagusamy, R. A Complete ranking of incomplete interval information. Expert Syst. Appl. 2014, 41, 1947-1954. [CrossRef]

5. Bai, Z.Y. An interval-valued intuitionistic fuzzy TOPSIS method based on an improved score function. Sci. World J. 2013, 2013, 879089. [CrossRef] [PubMed]

6. Garg, H. A new generalized improved score function of interval-valued intuitionistic fuzzy sets and applications in expert systems. Appl. Soft Comput. 2016, 38, 988-999. [CrossRef]

7. Nayagam, V.L.G.; Muralikrishnan, S.; Geetha, S. Multi-criteria decision-making method based on interval-valued intuitionistic fuzzy sets. Expert Syst. Appl. 2011, 38, 1464-1467. [CrossRef]

8. Sahin, R. Fuzzy multicriteria decision making method based on the improved accuracy function for interval-valued intuitionistic fuzzy sets. Soft Comput. 2016, 20, 2557-2563 [CrossRef]

9. Fangwei, Z.; Xu, S. Remarks to "Fuzzy multicriteria decision making method based on the improved accuracy function for interval-valued intuitionistic fuzzy sets". Soft Comput. 2017, 21, 2263-2268.

10. Nayagam, V.L.G.; Jeevaraj, S.; Dhanasekaran, P. An intuitionistic fuzzy multi-criteria decision-making method based on nonhesitance score for interval-valued intuitionistic fuzzy sets. Soft Comput. 2018, 21, 7077-7082. [CrossRef]

11. Jeevaraj, S. Similarity measure on interval valued intuitionistic fuzzy numbers based on non-hesitance score and its application to pattern recognition. Comp. Appl. Math. 2020, 39, 212. [CrossRef]

12. Pamucar, D.; Deveci, M.; Gokasar, I.; Işık, M.; Zizovic, M. Circular economy concepts in urban mobility alternatives using integrated DIBR method and fuzzy Dombi CoCoSo model. J. Clean. Prod. 2021, 323, 129096 [CrossRef]

13. Deveci, M.; Torkayesh, A.E. Charging Type Selection for Electric Buses Using Interval-Valued Neutrosophic Decision Support Model. IEEE Trans. Eng. Manag. 2021. [CrossRef]

14. Zhang, H.; Yu, L. MADM method based on cross-entropy and extended TOPSIS with interval-valued intuitionistic fuzzy sets. Knowl Based Syst. 2012, 30, 115-120. [CrossRef]

15. Hwang, C.L.; Yoon, K. Multiple Attribute Decision Making-Methods and Applications; Springer: Berlin/Heidelberg, Germany, 1981.

16. Peng, X.; Yang, Y. Fundamental properties of interval-valued Pythagorean fuzzy aggregation operators. Int. J. Intell. Syst. 2016, 31, 444-487. [CrossRef]

17. Jeevaraj, S. Ordering of interval-valued Fermatean fuzzy sets and its applications. Expert Syst. Appl. 2021, 185, 1-20. 